This is a pre-print version of the article

V.A. Fernandes and D.S.A. De Focatiis (2014), The role of deformation history on stress relaxation and stress memory of filled rubber, Polymer Testing 40, pp 124-132.

DOI: 10.1016/j.polymertesting.2014.08.018

The post-print can be found at http://dx.doi.org/10.1016/j.polymertesting.2014.08.018

\title{
The role of deformation history on stress relaxation and stress memory of filled rubber
}

\author{
Vanessa A. Fernandes, Davide S.A. De Focatiis * \\ Division of Materials, Mechanics and Structures, University of Nottingham, NG7 2RD, \\ Nottingham, United Kingdom \\ *Corresponding author: Tel.: +44 (0)115 95 14097; fax: +44 (0)115 9514115. \\ E-mail address: davide.defocatiis@ nottingham.ac.uk (D. S. A. De Focatiis)
}

\begin{abstract}
Although the magnitudes of inelastic and viscoelastic effects in filled rubbers are small relative to that of the elastic response, these effects are nevertheless critical in applications such as gaskets, seals and dampers. This study investigates the role of deformation history on relaxation of rubber through time-dependent experiments following a range of deformation histories. Two grades of carbon-black filled EPDM were subjected to uniaxial tensile deformation followed by stress-relaxation or stress memory at fixed deformation. Stress relaxation was found to be highly dependent on strain levels following a single loading. When an additional load-unload cycle was added to the history, the rubbers relaxed an approximately constant fraction of stress after a given time, provided that the strain at stress relaxation was smaller than the historical maximum. This fraction was independent of both the applied strain and of the maximum strain, and suggests that the relaxation process is independent of scragging procedures used to control the modulus. Stress memory observed following load-unload cycles was also approximately independent of strain history.
\end{abstract}

Keywords: Viscoelasticity, Mullins effect, EPDM, time-dependence, stress memory, stress relaxation.

\section{Introduction}

Elastomers are best known for their ability to undergo large elastic deformations, but there are many applications whose performance is dictated by the magnitude of inelastic and viscoelastic effects. For example, EPDM rubber is frequently employed in the production of seals and gaskets, and their performance is regularly evaluated in terms of compression set, defined as the degree to which a rubber remains permanently deformed when unloaded following an extended period of loading [1,2]. This reduced recovery can lead to failure in seals, and to reduced dissipation and functionality in dampers. Rubbers are inherently viscoelastic materials, however, and it can be inappropriate to talk about permanent deformation without knowledge of the response over longer timescales than those for which 
the material is observed. In fact, what is critical in both sealing and damping applications is precisely the time-dependence of the elastic recovery, although the timescales of interest may differ dramatically across different applications.

It is widely recognised that both the constitutive response and the viscoelastic behaviour of a rubber product are influenced by aspects such as choice of formulation, curing time and curing agents, and filler type and shape [3,4]. For example, it is well known that an increased filler fraction leads to both a stiffer response and to a greater degree of hysteresis in rubbers $[4,5]$. Deformation history plays an important role in the mechanical response of elastomers, best known through the manifestation of the softening phenomenon studied by Mullins and co-workers in the 1950s, and known as the Mullins effect [6,7]. In the Mullins effect, deformation causes (semi) permanent changes in the elastomer's microstructure that influence the mechanical response of the elastomer during subsequent deformations. As a consequence, a smaller stress is required to generate a given deformation in subsequent loadings, as long as the original deformation is not exceeded. If the deformation exceeds the historical maximum, the stress needed to deform the material becomes independent of this history, and returns to a value close to that needed to deform a virgin specimen to the same strain $[4,8]$.

In product applications whose performance is a strong function of the inelastic response, it is an important consideration to ask whether and how the degree of inelasticity is affected by deformation history. Rubber products are regularly scragged prior to being brought into service, by subjecting them to a deformation typically greater than that expected in service [9-11]. This process is normally intended to regulate the stiffness of rubber products. By the same process, some rubber products may need to be replaced if overloaded in such a way as to move the stiffness outside acceptable bounds. However, it is not obviouswhether and how the viscoelastic nature of the material is affected by scragging, and if overloads might require the rubber part to be replaced due to changes, not to stiffness, but to viscoelastic properties. The answer to this question lies in the relationship between the viscoelastic response and the deformation history.

Relatively few studies have focused on the viscoelastic response of rubber. Siouris and coworkers developed a method to record stress relaxation in elastomeric o-rings for gas turbines, focusing on the effects of lubricants and temperature [12], but did not explore the role of deformation history. Farzaneh et al. reported stress relaxation and recovery in polyurethane elastomers, focusing on the role of temperature in shape-memory applications [13]. Several studies employed an interrupted loading technique to attempt to reach an equilibrium elastic response of rubber, e.g. see $[14,15]$ but, in general, the response during relaxation in not the focus of such experiments.

A previous study carried out by De Focatiis et al. focused on cyclic uniaxial and biaxial deformation of EPDM rubber, suggested a means by which the viscous contribution to the response could be extracted from the loading and unloading parts of a cycle [16]. Once permanent set had been accounted for, the viscous contribution during constant rate deformation appeared relatively insensitive to both the strain level during the deformation and the maximum strain reached. This was in stark contrast to the elastic contribution, which varied markedly with both current and maximum strain, in accordance with the Mullins effect. The question as to whether and how the time-dependent response during stressrelaxation and recovery might also depend on deformation history appears, to the authors' knowledge, to have remained unanswered to date. With this in mind, the present paper reports experimental measurements of short term stress-relaxation and recovery on rubber 
subjected to a wide array of prior deformation histories, in part inspired by the ingenious set of stress-memory experiments on polymer glasses of Caruthers and co-workers [17], in order to shed light on this relatively unexplored phenomenon. The objective is to contribute to the body of knowledge necessary for a fully time- and history-dependent constitutive model of rubber deformation.

\section{Materials and methods}

\subsection{Material preparation}

Two grades of ethylene-propylene-diene rubber were studied in this work. The first is a sulphur cross-linked oil extended carbon black filled (nominally 50phr) EPDM, denoted EPDM1, and kindly provided by Dr T. Alshuth from the DIK. Sheets of $\sim 0.5 \mathrm{~mm}$ in thickness were cross-linked by compression moulding into $150 \mathrm{~mm} \times 150 \mathrm{~mm}$ flash moulds using a Daniels heated press at $160^{\circ} \mathrm{C}$ for 13 minutes [18]. The second is a carbon black filled EPDM provided pre- vulcanised in large $\sim 0.5 \mathrm{~mm}$ thick sheets by J-Flex Rubber Products, and denoted as EPDM2.

\subsection{Physical characterisation}

The densities of both materials after the vulcanisation process were measured using a Mettler Toledo XS105 analytical balance fitted with density kit, using deionised water as the medium, and rectangular specimens of mass $\sim 0.23 \mathrm{~g}$. The averages of nine repeats $( \pm 1 \mathrm{SD}$ ) are reported in Table 1. Thermal analysis was carried out using a TA Instrument DSC Q10 differential scanning calorimeter (DSC). Cured samples of $\sim 8 \mathrm{mg}$ were first heated to $140^{\circ} \mathrm{C}$ at a rate of $20^{\circ} \mathrm{C} \mathrm{min}{ }^{-1}$ to erase any thermal history, then cooled to $-75^{\circ} \mathrm{C}$ and reheated to $300^{\circ} \mathrm{C}$ at the same temperature rate, all in a $\mathrm{N}_{2}$ atmosphere. The glass transition temperatures, $T_{\mathrm{g}}$, were determined using TA Universal Analysis software as the mid-point of the temperature inflection using three tangent lines, and the averages of three repeats ( $\pm 1 \mathrm{SD})$ are reported in Table 1.

On the basis of equilibrium swelling experiments, an average molar mass of chains between cross-links, $M_{\mathrm{c}}$ was determined for both materials. Specimens of dimensions $\sim 20 \mathrm{~mm} \times 20$ $\mathrm{mm} \times 0.5 \mathrm{~mm}$ were immersed in analytical reagent grade toluene (from Fisher Scientific supplier) for 48 hours at room temperature $\left(20 \pm 1^{\circ} \mathrm{C}\right)$. The change in mass due to swelling was recorded with a Mettler Toledo XS105 analytical balance and used to obtain the volumetric fraction of rubber. Assuming tetrafunctional cross-links and using the FloryRehner equation [19], the average molar mass between cross-links $\left(M_{\mathrm{c}}\right)$ and the cross-link density, $\rho_{\mathrm{x}}$, based on three repeats $( \pm 1 \mathrm{SD})$ are reported in Table 1 .

A Shore A durometer was employed according to BS ISO 7619-1 to measure the hardness, $H$, of both materials. The average of three measurements $( \pm 1 \mathrm{SD})$ is reported in Table 1. An independent approximation of the carbon black filler content was determined using thermogravimetric analysis (TGA) with a TA Instrument SDT Q600. Samples of both grades of EPDM were first heated to $550^{\circ} \mathrm{C}$ at a rate of $10^{\circ} \mathrm{C} \mathrm{min}^{-1}$ under a $\mathrm{N}_{2}$ atmosphere. After cooling to $330^{\circ} \mathrm{C}$ at the same rate, the atmosphere was changed from inert to oxidative (air), and the samples heated to $800^{\circ} \mathrm{C}$ at a rate of $10^{\circ} \mathrm{C} \mathrm{min}$. Three weight loss fractions can be distinguished during the heating, associated with oils, rubber and carbon black. The final relative weight loss, associated with carbon black oxidation, is used as an indicator of filler content, $\varnothing_{\mathrm{c}}^{\mathrm{TGA}}$, expressed as parts per hundred rubber (phr) based on three measurements $( \pm$ 1SD) in Table 1. 
Table 1. Physical properties of EPDM1 and EPDM2.

\begin{tabular}{ccccccc}
\hline Material & $\begin{array}{c}\rho \\
\left(\mathbf{g ~ c m}^{-3}\right)\end{array}$ & $\begin{array}{c}\boldsymbol{T}_{\mathrm{g}} \\
\left({ }^{\mathbf{C}}\right)\end{array}$ & $\begin{array}{c}\boldsymbol{M}_{\mathbf{c}} \\
(\mathbf{D a})\end{array}$ & $\begin{array}{c}\boldsymbol{\rho}_{\mathrm{x}} \\
\left(\mathbf{m o l ~ m}^{-3} \times \mathbf{1 0}^{-4}\right)\end{array}$ & $\begin{array}{c}\boldsymbol{H} \\
(\text { Shore A) }\end{array}$ & $\begin{array}{c}\phi_{\mathrm{c}}^{\mathrm{TGA}} \\
(\mathbf{p h r})\end{array}$ \\
\hline $\boldsymbol{E P D M 1}$ & $1.03 \pm 0.01$ & $-52.0 \pm 1.5$ & $1098 \pm 49$ & $4.56 \pm 0.19$ & $50 \pm 1$ & $35.5 \pm 0.4$ \\
\hline $\boldsymbol{E P D M 2}$ & $1.14 \pm 0.01$ & $-47.4 \pm 1.0$ & $1965 \pm 11$ & $2.54 \pm 0.01$ & $60 \pm 1$ & $64.3 \pm 0.3$ \\
\hline
\end{tabular}

\subsection{Mechanical characterisation}

Mechanical test specimens were cut from sheet using a hand-operated Wallace specimen cutting press fitted with a dumbbell shape cutter type 1BA according to BS ISO 527-2. Individual specimen cross-sections were measured using a Hildebrand rubber thickness gauge according to ISO 23529 in the thickness direction, and a calibrated scanner system in the width direction. Uniaxial mechanical deformation was carried out using an Instron 5969 tensile testing machine equipped with $50 \mathrm{~N}$ load cell and an Instron counterbalanced travelling extensometer at room temperature $\left(20 \pm 1^{\circ} \mathrm{C}\right)$, at constant cross-head speed corresponding to a nominal strain rate of $0.1 \mathrm{~s}^{-1}$. In order to study the influence of deformation history on stress relaxation, four test protocols (TPs) were applied prior to recording stress relaxation at fixed displacement for a further $600 \mathrm{~s}$. The test protocols TP1TP4 are shown schematically in Figure 1 as strain as a function of time, and consist of the following operations:

TP1. Specimens subjected to a single loading ramp through to strains $\varepsilon_{\max }$ ranging between 0.5 and 2.5 for EPDM1, and between 0.5 and 5 for EPDM2, followed by stress-relaxation at constant strain for a further $600 \mathrm{~s}$.

TP2. Specimens subjected to a single load-unload cycle to a maximum strain $\varepsilon_{\max }=1,1.5$ and 2 for EPDM1, and of $\varepsilon_{\max }=1,2$ and 4 for EPDM2, unloading to $0.1 \mathrm{~N}$ (to prevent buckling of the specimen), followed by a single loading ramp through a range of strains $\varepsilon_{\text {end }}$ between 0.5 and 2.5 for EPDM1, and between 0.5 and 5 for EPDM2, followed by stress-relaxation at constant strain for a further 600s.

TP3. Specimens subjected to a single load-unload cycle to a maximum strain $\varepsilon_{\max }$ between 0.5 and 2 for EPDM 1, and between 0.5 and 4 for EPDM2, unloading to the strain corresponding to a force of $0.1 \mathrm{~N}$ (to prevent buckling of the specimen), followed by stress-relaxation at constant strain for a further $600 \mathrm{~s}$.

TP4. Specimens subjected to a single loading ramp through to $\varepsilon_{\max }=2$, unloaded to strains $\varepsilon_{\text {end }}^{*}$ ranging between 1.75 and 0.5 for both materials, followed by stressrelaxation at constant strain for a further 600 s.

In Figure 1, the symbols indicate the start of the relaxation stage, beginning at time $t_{0}$, where the stress at the start of the relaxation is denoted as $\sigma_{\text {end. }}$ The maximum strain reached during the test protocol is denoted as $\varepsilon_{\max }$, and the final strain, at the start of the stress relaxation, is denoted as $\varepsilon_{\text {end }}$ where the final strain rate is positive, and $\varepsilon_{\text {end }}^{*}$ where the final strain rate is negative. Each test was performed using a fresh, undeformed specimen. In total, 42 specimens were tested for EPDM1 and 35 for EPDM2. 

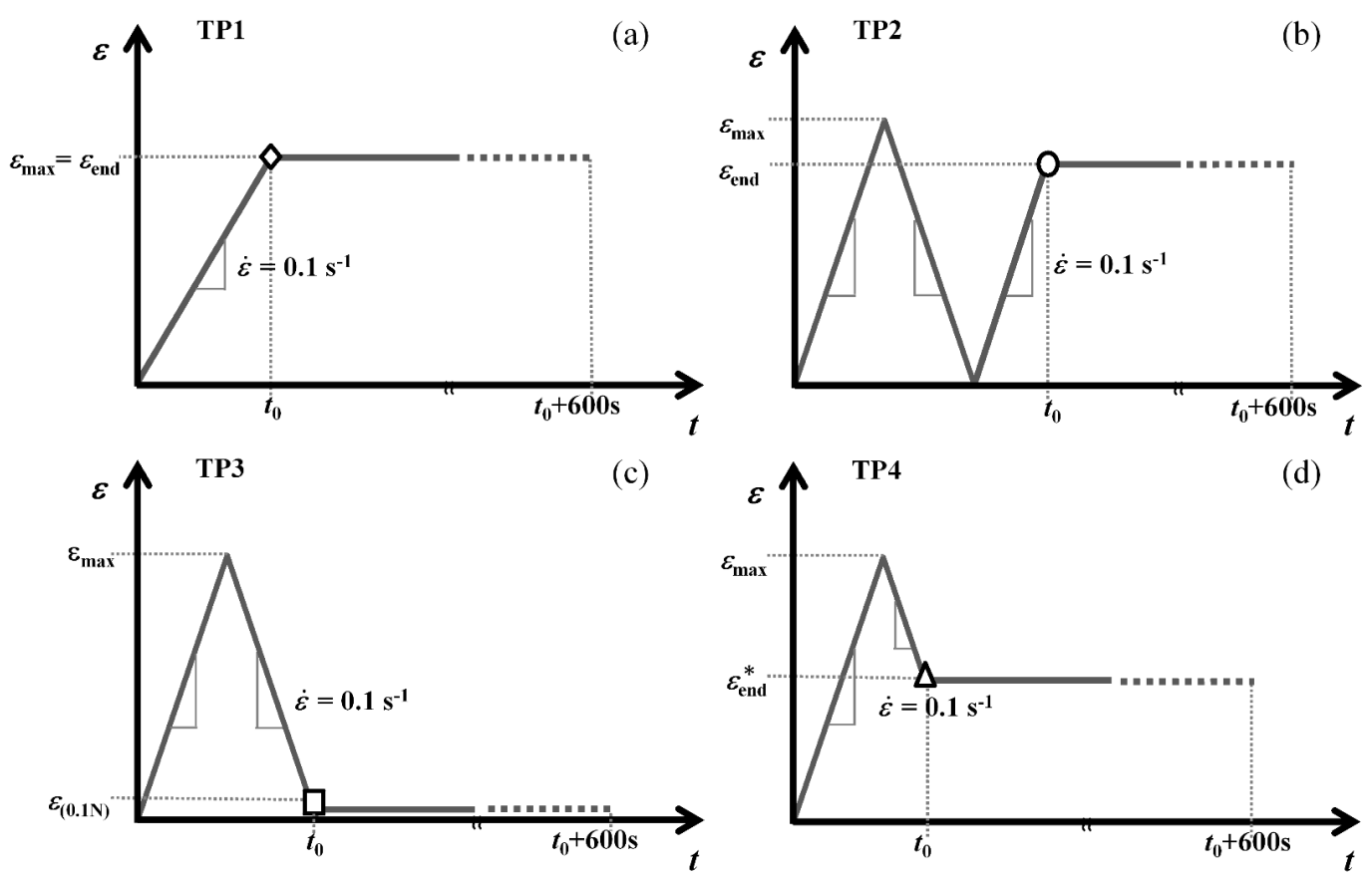

Fig 1. Strain histories imposed on specimens according to protocols (a) TP1, (b) TP2, (c) TP3 and (d) TP4. Symbols (diamond, circle, square and triangle) indicate the start of the stressrelaxation and stress-memory stages, at time $t_{0}$. The maximum strain reached during the test protocol is denoted as $\varepsilon_{\max }$, and the final strain, at the start of the stress relaxation, is denoted as $\varepsilon_{\text {end }}$ or $\varepsilon_{\text {end }}^{*}$ respectively, depending on whether the sign of the strain rate during the final stage of the load history is positive or negative.

Typical nominal stress vs strain responses for EPDM1 and EPDM2 indicating the specific points at which stress-relaxation and stress-memory measurements were started are shown in Figs 2 and 3 respectively.

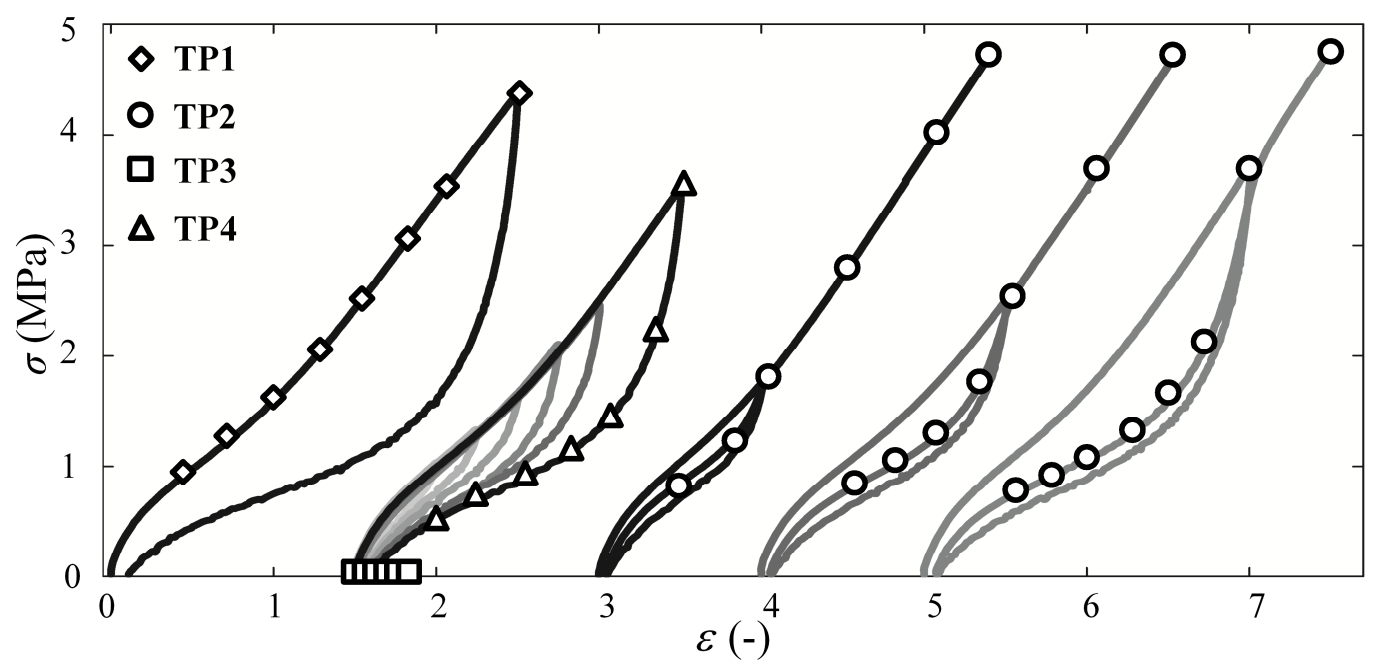

Fig 2. Nominal stress measured as a function of strain for EPDM1 specimens deformed according to TP1, TP4 (offset by $\varepsilon=1.5$ ), TP3 (offset by $\varepsilon=1.5$ ), and TP2 (offset by $\varepsilon=3,4$ 
and 5). Symbols indicate the positions of the start of the stress-relaxation and stress-memory measurements (data not shown here), where the stress is $\sigma_{\text {end. }}$.

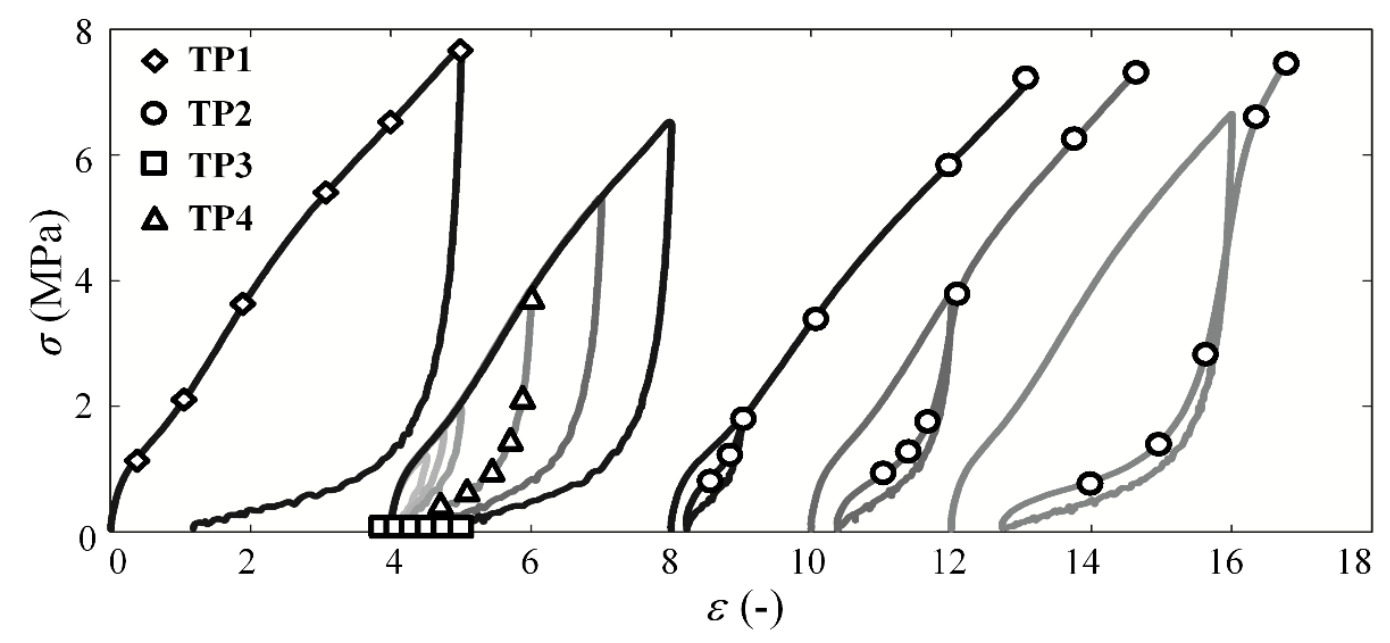

Fig 3. Nominal stress measured as a function of strain for EPDM 2 specimens deformed according to TP1, TP4, TP3 (offset by $\varepsilon=4$ ), and TP2 (offset by $\varepsilon=8,10$ and 12). Symbols indicate the positions of the start of the stress-relaxation and stress-memory measurements (data not shown here), where the stress is $\sigma_{\text {end. }}$.

\section{Results}

\subsection{Dependence of stress relaxation on deformation history}

The stress relaxation stages of specimens of EPDM1 and EPDM2 following TP1 are shown in Figs 4 (a) and (b) respectively, normalised with respect to the stress at the end of the loading ramp, $\sigma_{\text {end. }}$. The response is significantly dependent on strain history, with a bigger fraction of the stress relaxing following loading to larger strains. In both materials there is a clear dependence of stress on the maximum strain applied. EPDM2 relaxes a larger fraction of the stress than EPDM1 for a given strain history, consistent with its greater hardness and carbon black content. 

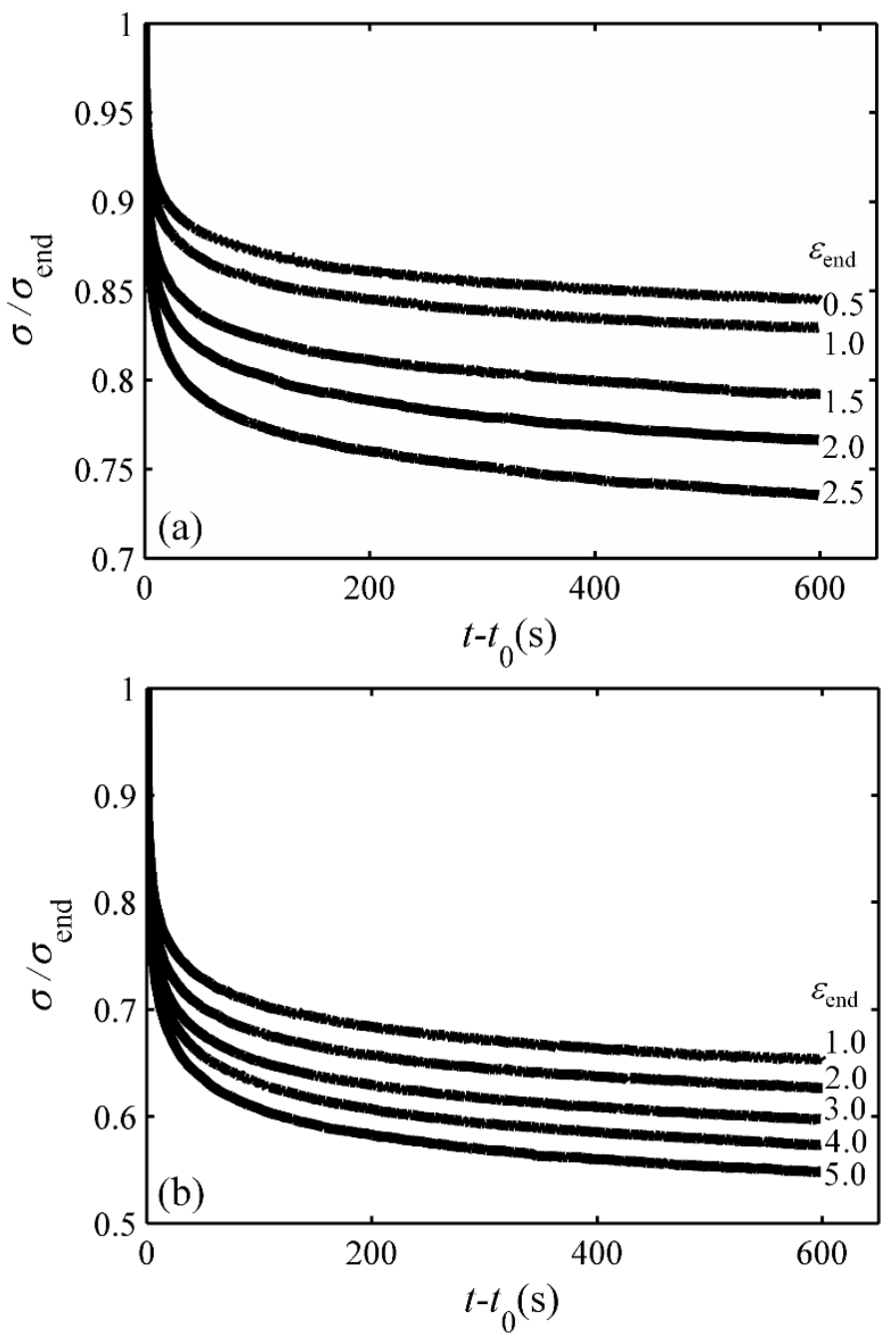

Fig 4. Normalised stress relaxation $\sigma / \sigma_{\text {end }}$ of EPDM1 (a) and EPDM2 (b) specimens subjected to a simple loading ramp to various strain levels $\varepsilon_{\text {end }}$ (as marked on the figure) according to TP1.

The normalised stress relaxation of EPDM1 and EPDM2 specimens subjected to TP2 is reported in Figs 5 (a) and (b), respectively. TP2 differs from TP1 by the inclusion of an additional load-unload cycle, illustrated here for the case where $\varepsilon_{\max }=2$. The stress relaxation response of both materials differs clearly from that of TP1 in Fig. 4, with most specimens relaxing a smaller fraction of the stress than their counterparts from TP1. The responses can be grouped into two parts: for the cases where $\varepsilon_{\text {end }}<1.75$, the fraction of stress relaxing

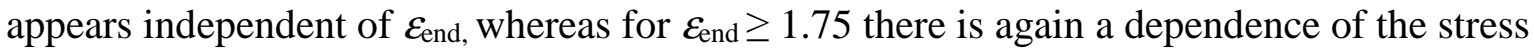
relaxation on the value of $\varepsilon_{\text {end }}$, and the relaxation becomes identical to that observed for the same corresponding $\varepsilon_{\text {end }}$ values in TP1 in Fig. 4 . It can be observed that a fixed fraction of stress relaxes, independently of strain level, if $\sigma_{\text {end }}$ is sufficiently smaller than $\sigma_{\max }$. 

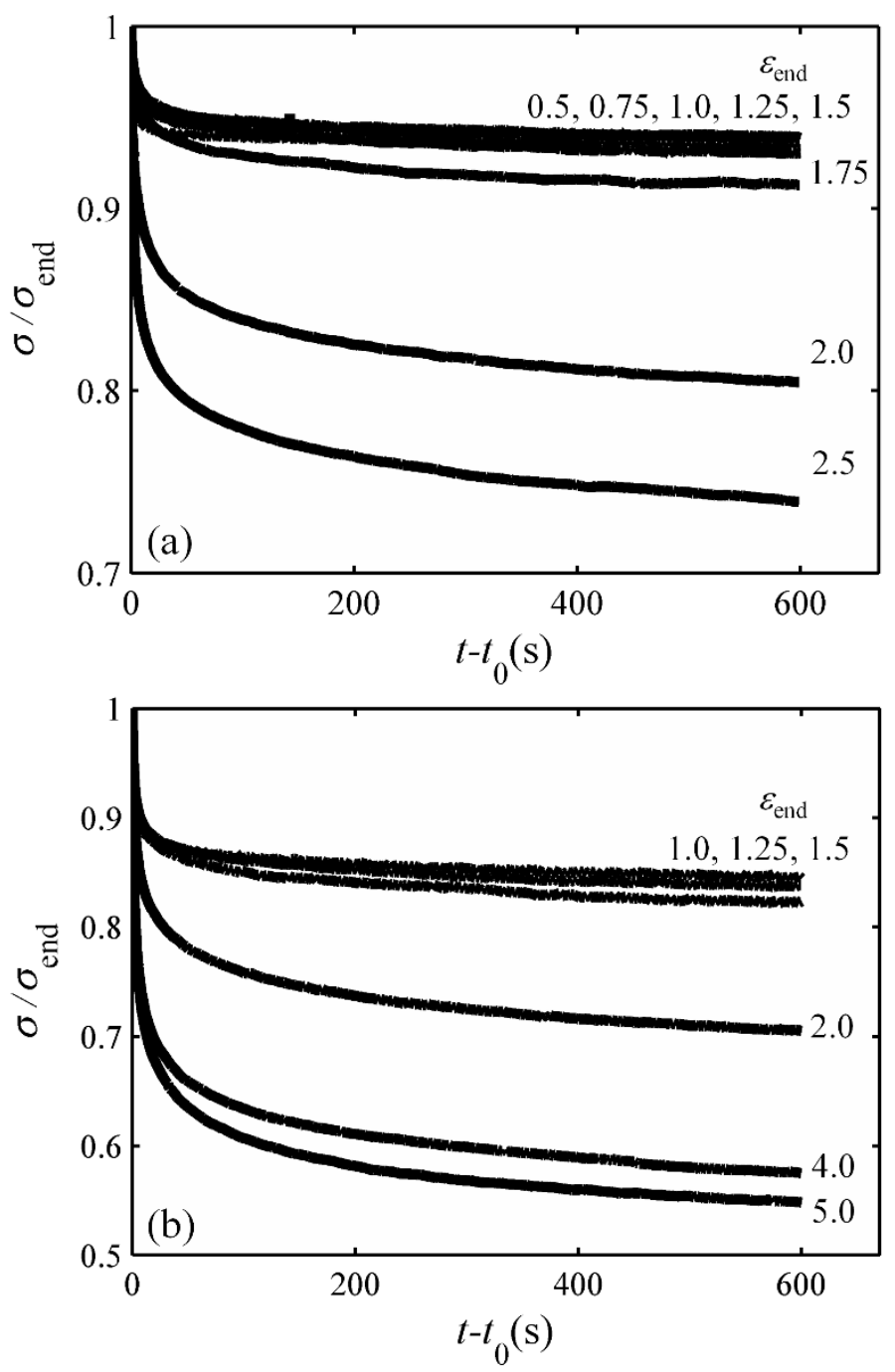

Fig. 5 Normalised stress relaxation $\sigma / \sigma_{\text {end }}$ of EPDM1 (a) and EPDM2 (b) specimens subjected to a load-unload ramp through to $\varepsilon_{\mathrm{max}}=2$, and reloaded to the strain levels $\varepsilon_{\text {end }}$ (marked on the figure), according to TP2.

\subsection{Dependence of stress memory on deformation history}

The stress-memory, or recovery of stress after unloading, is shown for EPDM1 and EPDM2 following a single load-unload cycle to varying levels of $\varepsilon_{\text {max }}$, according to TP3, in Figs. 6a and $6 \mathrm{~b}$, respectively. The stress memory and the magnitude of the stress present after $600 \mathrm{~s}$, is remarkably similar for all specimens within each material, and appears to be largely independent of the deformation history, i.e. of the fact that specimens experienced dramatically differing stresses during the load-unload cycle prior to the memory experiment (e.g. see Fig. 2, TP3). This is in sharp contrast with the dependence of stress relaxation on history observed in TP1 and TP2. The only exception to this is the case where $\varepsilon_{\max }=0.5$ in EPDM2. The greater filler content of EPDM2 results in a larger permanent set, and hence a shorter unloading phase, and as such it may be surmised that the stress memory has insufficient time to build up in this particular example. 

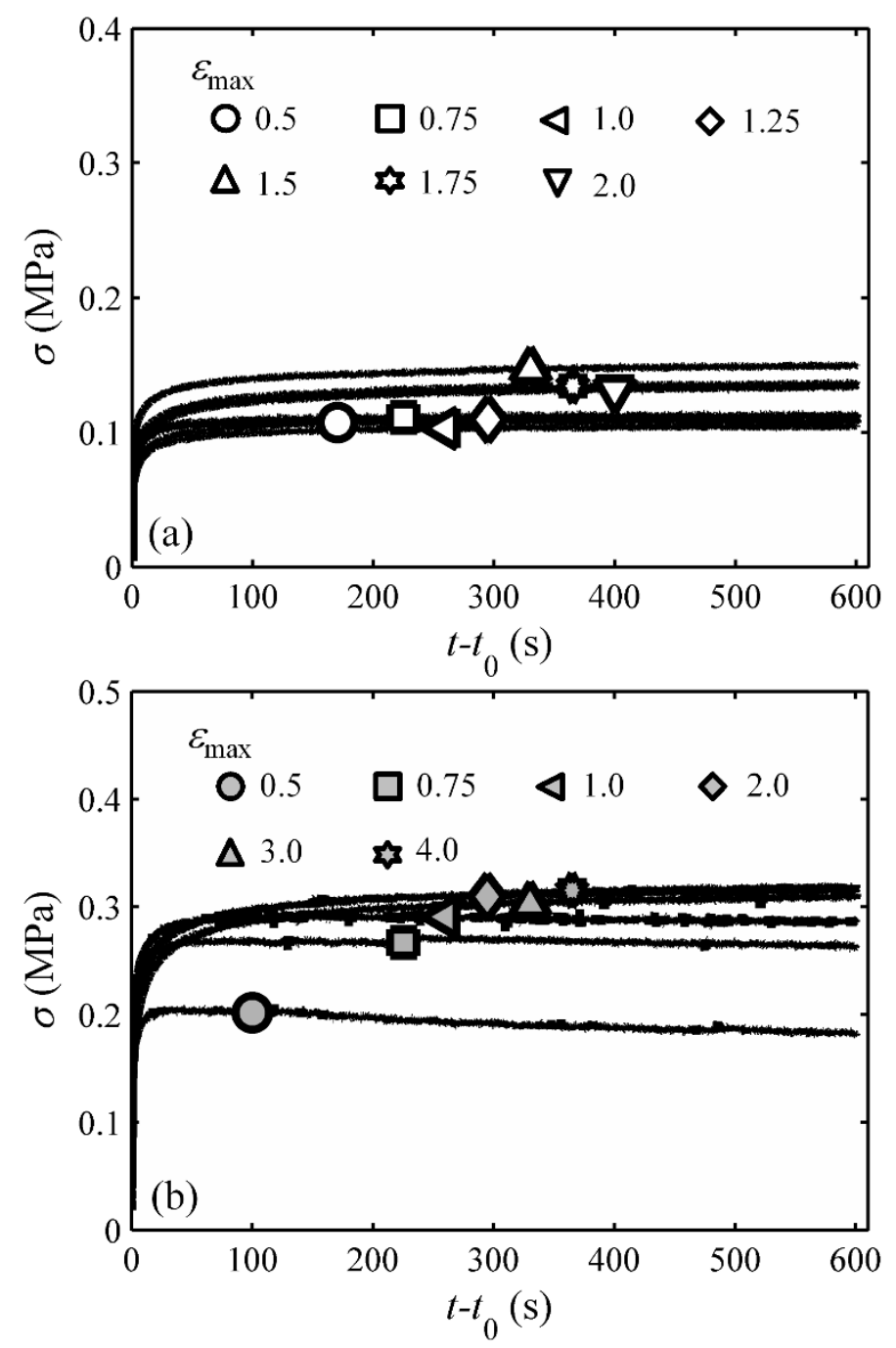

Fig. 6 Recovery of stress, or stress-memory, $\sigma$, of EPDM1 (a) and EPDM2 (b) specimens subjected to a single load-unload ramp through to $\varepsilon_{\max }$ (marked on the figure), unloaded to 0.1 $\mathrm{N}$ and held at constant displacement, according to TP3.

\subsection{Stress relaxation and memory following partial unloading}

The time-dependent stress $\sigma$ normalised with respect to the stress at the $e n d$ of the relaxation phase $\sigma_{\mathrm{r}, 600}$, is shown for specimens deformed according to TP4, deformed to $\varepsilon_{\max }=2$ and subsequently partially unloaded, in Figs 7 (a) and (b) for EPDM1 and EPDM 2, respectively. The insets show the initial stages of the time-dependence in more detail. Here, stress relaxation can be observed for unloading to $\varepsilon_{\text {end }}^{*}=1.5$, and of stress recovery for unloading to smaller strains, between $\varepsilon_{\text {end }}^{*}=1.25$ and 0.5 , as well as for unloading to $0.1 \mathrm{~N}$ (corresponding to $\varepsilon_{\text {end }}^{*}$ of $\sim 0.09$ for EPDM1 and $\varepsilon_{\text {end }}^{*} \sim 0.38$ for EPDM2). There is a striking difference relative to the data from TP2 shown in Fig. 5 where, upon reloading to the same strains, there is always stress relaxation, even at $\varepsilon_{\text {end }}=0.5$, and never stress recovery. 

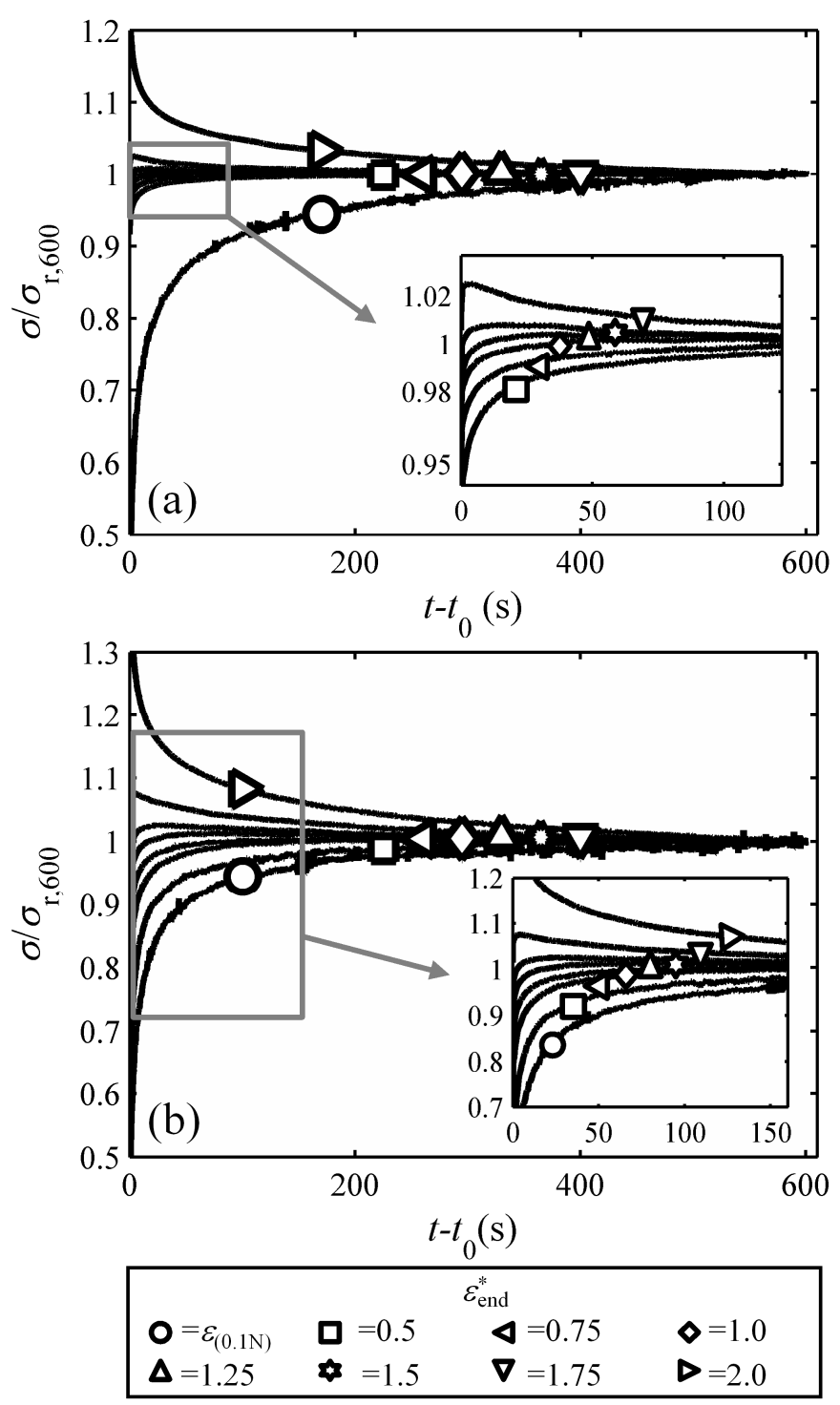

Fig. 7 Time-dependent stress $\sigma$ normalised with respect to the value at the end of the relaxation, $\sigma_{\mathrm{r}, 600}$, as a function of time of EPDM1 (a) and EPDM2 (b) specimens subjected to a loading ramp through to $\varepsilon_{\max }=2$, unloaded to the strain levels $\varepsilon_{\text {end }}^{*}$ (marked on the figure), according to TP4.

\section{Discussion}

\subsection{Stress relaxation}

The time-dependent responses of both grades of EPDM to stress-relaxation are clearly nonlinear viscoelastic when subjected to a single loading ramp, according to TP1 (Fig. 4). When a load-unload ramp is inserted prior to this, according to TP2 (Fig. 5), the responses can be separated into two parts, with a transition region in between. On the one hand, where the reloading strain $\mathcal{E}_{\text {end }}$ is larger than the maximum strain reached in the first load-unload ramp $\varepsilon_{\max }$, the stress-relaxation is identical to that observed following a single ramp. This suggests that, when the maximum strain experienced in the previous ramp (related to the scragging process) is smaller than the strain at which the stress-relaxation occurs, the relaxation of the 
material is unaffected by this historical ramp. The same type of behaviour is observed in the stress during the loading ramp, in accordance with the Mullins effect. On the other hand, where the reloading strain $\varepsilon_{\text {end }}$ is sufficiently smaller than the maximum strain reached in the first load-unload ramp $\varepsilon_{\max }$, the stress-relaxation becomes linear viscoelastic, i.e. the fraction of stress relaxing at a given time is independent of the magnitude of the applied stress. This is somewhat unexpected, especially at such large strains. For EPDM1, after $600 \mathrm{~s}$ of relaxation, this constant relaxing fraction of the stress is $\sim 0.06$, whereas for EPDM2 it is $\sim 0.17$, consistent with the greater filler content in EPDM2. There is a transition region corresponding to a reloading strain $\varepsilon_{\text {end }}$ equal to or slightly smaller than the maximum strain reached in the first load-unload $\operatorname{ramp} \varepsilon_{\max }$, where the response appears to lie somewhere between the two conditions.

The fraction of unrelaxed stress at $600 \mathrm{~s}, \sigma_{\mathrm{r}, 600} / \sigma_{\text {end, }}$, is plotted as a function of $\varepsilon_{\text {end }}$ for EPDM1 in Fig. 8, and for EPDM2 in Fig. 9. Additional experiments were carried out according to TP2, but with different load-unload strains $\varepsilon_{\max }$ of 1.0 and 1.5 for EPDM1, and of 1.0 and 4.0 for EPDM2. Data from TP1, i.e. with no previous loading ramp, is also shown in the figures.

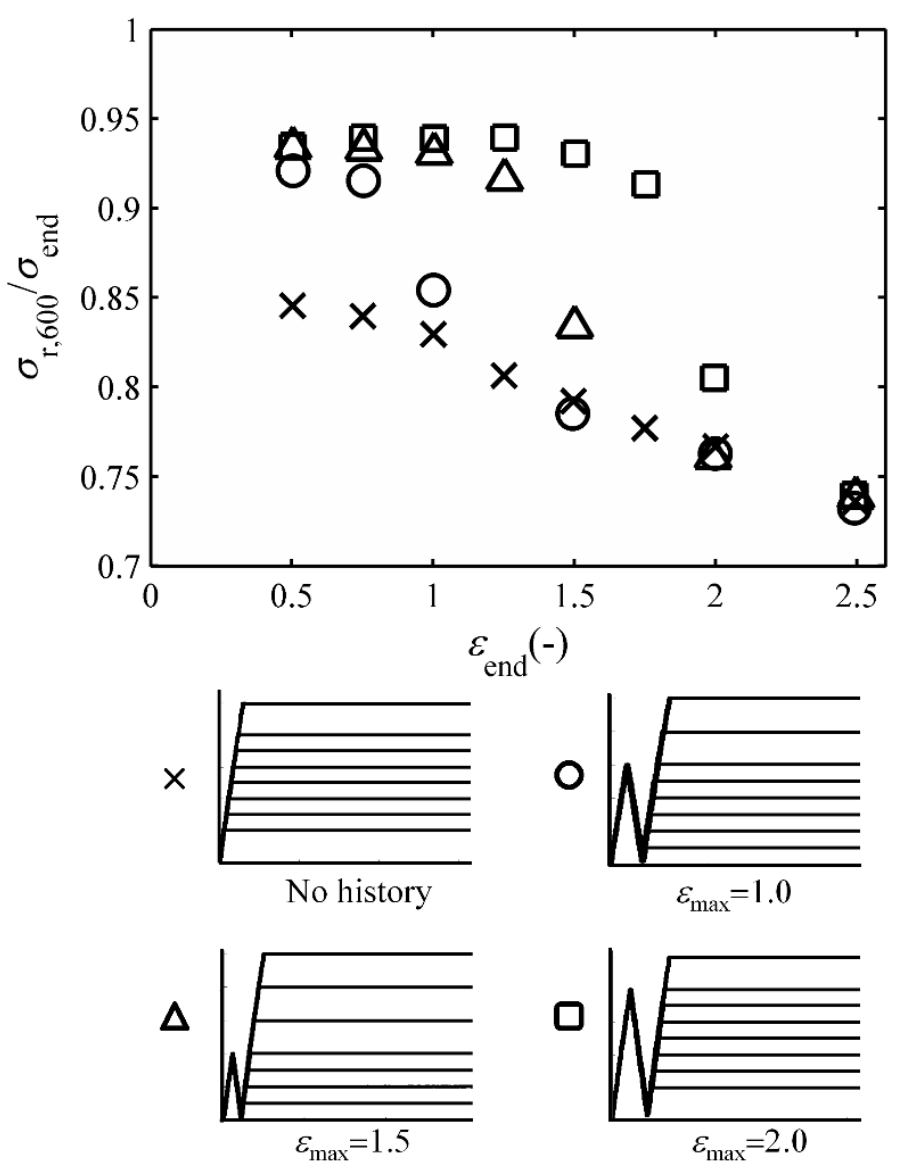

Fig. 8 Fraction of unrelaxed stress $\sigma_{\mathrm{r}, 600} / \sigma_{\text {end }}$ of EPDM1 subjected to deformation histories TP1 and TP2 to varying levels of maximum deformation $\mathcal{E}_{\max }$ (shown in the legend) and final deformation $\varepsilon_{\text {end }}$. 


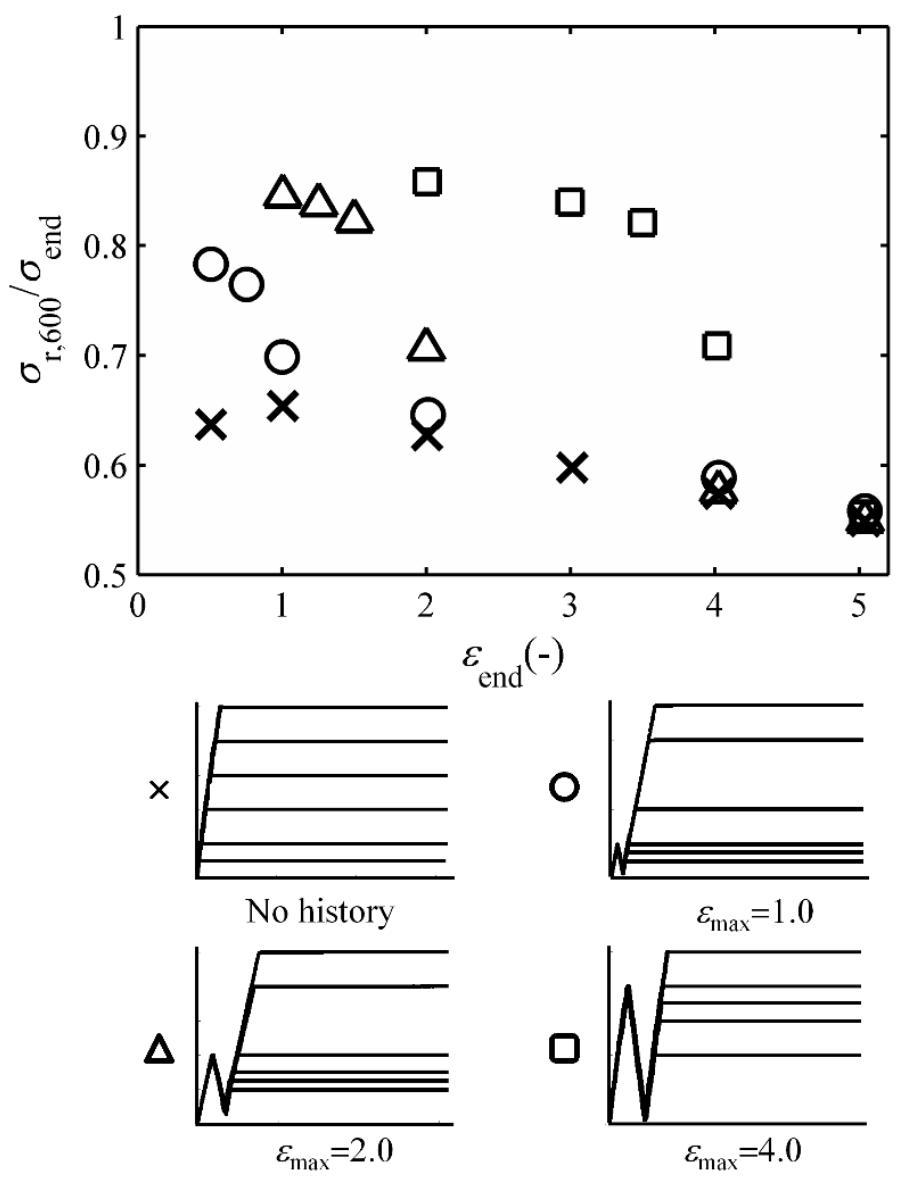

Fig. 9 Fraction of unrelaxed stress $\sigma_{\mathrm{r}, 600} / \sigma_{\text {end }}$ of EPDM2 subjected to deformation histories TP1 and TP2 to varying levels of maximum deformation $\varepsilon_{\max }$ (shown in the legend) and final deformation $\varepsilon_{\text {end }}$.

The data show a distinctive pattern. Where stress relaxation follows a single loading ramp (TP1), the unrelaxed fraction is significant, ranging between 0.76-0.85 for EPDM1 and between 0.55-0.67 for EPDM2. Specimens that have instead experienced a previous loadunload ramp, and that are reloaded to strains smaller than the previous maximum, exhibit an approximately constant fraction of unrelaxed stress, independent of both the reloading strain $\mathcal{E}_{\text {end }}$ (and hence the actual value of the stress $\sigma_{\text {end }}$ ) and of the previously reached maximum strain $\varepsilon_{\max }$. For EPDM1 this constant fraction of unrelaxed stress is $\sim 0.94$, whereas for EPDM2 it is $\sim 0.83$. In the transition regions the unrelaxed fraction moves from this constant value towards the value measured after a single loading ramp with increasing $\mathcal{E}_{\text {end. }}$. Fig.s 8 and 9 may be thought of as maps of the time-dependent equivalent of the pseudo-cyclic loadunload-reload curves frequently used to illustrate the Mullins effect [20].

The fact that the relaxed fraction appears to be independent of the reloading strain $\varepsilon_{\text {end }}$ (when this is sufficiently smaller the previous maximum $\varepsilon_{\max }$ ) is an indication that an appropriately scragged rubber behaves, and may be modelled as, a linear viscoelastic material. What may be less apparent is that the relaxed fraction is also independent of the value of the previous maximum $\varepsilon_{\max }$. This is in sharp contrast to the constitutive response during loading of the 
rubber, which is well known to be affected by the previous maximum strain reached [16], in the well-known Mullins effect, and can be observed in the present data in Fig. 2.

Since it is the formulation that controls the relaxing fraction, this finding is an important consideration for the design of components whose performance depends on the viscoelastic response. The phenomenon suggests that the degree of scragging can be safely used to tune the modulus, without unexpectedly affecting the viscoelastic response. The degree of relaxation can instead be controlled by the formulation itself. The two grades of EPDM used here differ most significantly in their cross-link densities and in their filler fractions. With the present data it is not possible to discern which of these parameters is the dominant one, although it would be reasonable to expect that both may influence the post-scragging relaxed fraction to some degree.

\subsection{Stress memory and combined effects}

TP3 has shown (in Fig. 6) that, following a load-unload cycle, the materials recover an approximately constant stress (within experimental error), independently of the magnitude of the maximum strain $\varepsilon_{\max }$ experienced within the first cycle. For EPDM1, after $600 \mathrm{~s}$, this is $\sim 0.1 \mathrm{MPa}$, and for EPDM3 it is $\sim 0.3 \mathrm{MPa}$. The stress-memory effect appears to be independent of the degree of scragging. This effect takes place as a result of a negative flow stress during the unloading stage, which leads to a state of self-stress when $\sigma_{\text {end }}$ reaches approximately zero and the cross-head is stopped (in practice $\sigma_{\text {end }}$ is $\sim+0.04 \mathrm{MPa}$, corresponding to a tensile force of $0.1 \mathrm{~N}$ for the cross-sections employed). The fact that a stress appears over time is due to the relaxation of this negative (compressive) flow stress which leaves behind a positive (tensile) elastic part. At these relatively small strains, there is little difference in the elastic response as a consequence of the different $\varepsilon_{\text {max }}$ values.

Therefore, this points to a flow stress that is constant and unaffected by $\varepsilon_{\text {max }}$, at least at these small strains.

It is worth reminding the reader that the relaxed fraction of stress in TP2 tests is equally unaffected by $\varepsilon_{\max }$. The difference between these tests is that in TP 2 tests a constant fraction of stress relaxes, and hence the magnitude of relaxing stress depends on the magnitude of the stress prior to relaxation, whereas in TP3 tests, a constant amount of stress relaxes. This could point to two manifestations of the relaxation process - one that is intrinsically coupled to the elastic stress, dominant when the rubber is elastically deformed, and another that can be observed when the rubber is elastically unloaded, and hence that is largely unaffected by $\varepsilon_{\max }$.

TP4 has shown that stress-memory can occur even when the rubber is not fully unloaded, as might be expected by a flow process. But it has also shown that, in some cases, stressrelaxation can occur even following unloading, in particular in the cases where the unloading is limited to smaller strains. This could be interpreted as a time- and strain-dependent relaxation process associated with the Mullins effect itself. As the material nears the historical maximum stress, this relaxation process is activated such as to reduce the elastic stiffness in a quasi-permanent manner that leads to the Mullins effect. In TP1, this process is taking place in every test, but in TP2 it only contributes where the stress (or strain) is sufficiently large compared with the historical maximum.

\subsection{Implications for constitutive modelling}

There are several patterns emerging from the data presented, and each of these contributes to shaping the simplest form of constitutive model that is consistent with the observed time- and 
history-dependence. Here we focus on the most striking of these: the fact that when rubber is stretched to a deformation that is within (and not too close to) the maximum previously reached strain, a constant fraction of the stress relaxes, independent of both current and historical strains. This implies that the relaxation process must be coupled to the elastic contribution such that (for a pre-scragged rubber) a fixed fraction of the network relaxes independently of the constitutive response of the elastic network. It is well known that the variation of the constitutive response of the elastic network (associated with the microstructural changes underpinning the Mullins effect) is affected by strain history, but any model formulation should ensure that this change in network elasticity does not affect the linear viscoelastic nature of the material.

As an illustrative 1-D example, consider a Standard Linear Solid type model, widely used in the modelling of the rate-dependent response of elastomers [15, 21, 22] and polymers [23]. What is required is to couple the spring stiffnesses of the elastic and viscoelastic parts through a parameter, denoted as $r$, which is associated with the fraction of relaxing stress at long times, as shown in Fig. 10. Here the spring stiffness $(1-r) G$ is intended to represent the elastic response, and that marked $r G$ the elastic part of the Maxwell element that gives rise to the relaxation. Irrespective of the nature of the springs, and of the viscosity of the dashpot $\eta$, a constant fraction $r$ of stress will eventually relax following a loading, provided that the Weissenberg number of the process (the product of strain rate and relaxation time) is sufficiently larger than unity. The Mullins effect may be brought into the model through an evolution equation for $G$. This evolution, associated with the Mullins effect, and associated with the scragging process would leave the relaxing fraction unaffected, as exhibited in the experimental data. Several forms of this type of evolution have been proposed in the literature [8], and there is good experimental evidence to support this approach [16]. It is likely, however, that a generalised Maxwell model formulation will be required to capture the intricacies of the relaxation spectrum. Work is on-going in our laboratory to assemble a fully 3-dimensional time- and history-dependent constitutive model able to represent all of the phenomena observed here, but achieving good agreement with different types of timedependent experimental data with physically-based material parameters remains an on-going challenge for the rubber community [24].

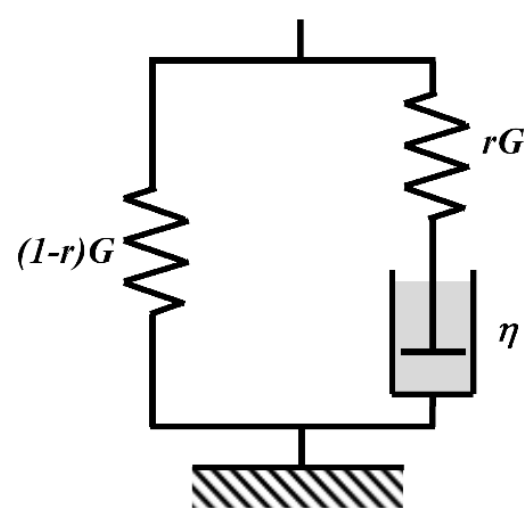

Fig. 10 Schematic diagram of a one-dimensional model with coupled elastic and viscoelastic arms.

\section{Conclusions}

This study has presented new experimental measurements of stress-relaxation and stressmemory on two grades of EPDM rubber. Specimens were subjected to a variety of carefully 
constructed deformation histories in order to elucidate the underlying mechanisms of timedependent stress evolution.

When stress relaxation followed a simple loading ramp in virgin rubber, the fraction of stress relaxing was highly strain-dependent. Instead, when stress relaxation was carried out following a prior load-unload cycle, to a strain smaller than the previous maximum, a constant fraction of the stress relaxed, independent of both the applied strain and of the previous maximum strain. This fraction varied with the different rubbers, and was larger in the more highly cross-linked EPDM rubber with the higher filler content. This has important industrial implications since it means that the relaxation remains independent of any preparatory scragging procedures, and that scragging may be safely used to control the modulus of rubber products without affecting the relaxation process. Maps of unrelaxed fraction as a function of applied strain for different histories were presented, and can be interpreted as the time-dependent equivalent of pseudo-cyclic load-unload curves commonly used to summarise the Mullins effect. These maps should prove useful to the community interested in predicting the relaxation of EPDM rubber products following complex strain histories, as well as to developers of constitutive models for rubber accounting for timedependence and the Mullins effect.

Stress memory was recorded following load-unload cycles, and was found to be approximately independent of strain history. Both stress relaxation and stress memory were observed over time after a partial unloading, but only stress relaxation was observed after partial loading. This, and the other effects observed, suggests that a time-dependent constitutive model may be able to be formulated with a decoupled Mullins evolution of the elastic part of the response and a separate viscoelastic relaxation process. The experiments have confirmed that deformation history can have, under certain conditions, a significant and well-defined role in the determination of the time-dependent response of rubber.

\section{Acknowledgements}

The authors gratefully acknowledge the contribution of Dr. T. Alshuth of the German Institute of Rubber Technology (DIK) in supplying the EPDM1 compound, and of J-Flex Rubber Products in providing the EPDM2 sheet. VAF acknowledges Dean of Engineering funding from the University of Nottingham.

\section{References}

[1] J.W. Alistair Hill, Stress relaxation and compression set behaviour of thermoplastic elastomers in sealing applications., Thermoplastic Elastomers, III (1991) 14.

[2] P. Tuckner, Compression stress relaxation test comparisons and development, SAE Technical Paper. SAE World Congress, (2000).

[3] T. Vijayaram, A technical review on rubber, Int J Des Man Tech, 3 (2009) 13.

[4] C.P. Buckley, Experimental methods for rubberlike solids, in: G. Saccomandi and R.W. Ogden (Ed.s) Mechanics and Thermomechanics of Rubberlike Solids, Springer, Vienna, 2004.

[5] H.M. Osman, S.A.A. Ghani, T.M. Madkour, A.R. Mohamed, Stress relaxation in carbon black loaded butyl rubber, J Appl Polym Sci, 77 (2000) 1067-1076.

[6] L. Mullins, Effect of stretching on the properties of rubber, J Rubb Res, 16 (1947) 22.

[7] L. Mullins, M. Tobin, Theoretical model for the elastic behavior of filler-reinforced vulcanized rubbers, Rubb Chem Tech, 30 (1957) 555-571. 
[8] J. Diani, B. Fayolle, P. Gilormini, A review on the Mullins effect, Eur Polym J, 45 (2009) 601-612.

[9] J.G.R. Kingston, A.H. Muhr, Effect of scragging on parameters in viscoplastic model for filled rubber, Plast Rub Comp, 40 (2011) 161-168.

[10] J. Kingston, The effect of scragging strain on the dynamic mechanical properties of filled rubber, J Rubb Res, 11 (2008) 1-12.

[11] BS ISO 4664:1998. Rubber: guide to the determination of dynamic properties, British Standard Institution, Geneva, 1998.

[12] S. Siouris, B. Shaw, C. Wilson, Method for the evaluation of elastomeric seals by compression stress relaxation, Polym Test, 32 (2013) 1299-1305.

[13] S. Farzaneh, J. Fitoussi, A. Lucas, W. Bocquet, A. Tcharkhtchi, Shape memory effect and properties memory effect of polyurethane, J Appl Polym Sci, 125 (2013).

[14] P. Haupt, S. K., Viscoplasticity of elastomeric materials: experimental facts and constitutive modelling, Arch Appl Mech, 71 (2001) 20.

[15] J.S. Bergstrom, M.C. Boyce, Large strain time-dependent behavior of filled elastomers, Mech Mater, 32 (2000) 627-644.

[16] D.S.A. De Focatiis, F. Abraham, C.P. Buckley, Multi-axial viscoelastic deformation of carbon-black filled EPDM rubber, Constitutive Models for Rubber VI, (2010) 187-192.

[17] J.W. Kim, G.A. Medvedev, J.M. Caruthers, The response of a glassy polymer in a loading/unloading deformation: The stress memory experiment, Polymer, 54 (2013) 59936002.

[18] D.S.A. De Focatiis, Tooling for near net-shape compression moulding of polymer specimens, Polym Test, 31 (2012) 550-556.

[19] P.J. Flory, Principles of Polymer Chemistry, Ithaca: Cornell University Press, USA, 1953.

[20] D.S.A. De Focatiis, D. Hull, A. Sanchez-Valencia, Roles of prestrain and hysteresis on piezoresistance in conductive elastomers for strain sensor applications, Plast Rubber Compos, 41 (2012) 9.

[21] A. Plaseied, A. Fatemi, Deformation response and constitutive modeling of vinyl ester polymer including strain rate and temperature effects, J Mater Sci, 43 (2008) 1191-1199.

[22] H. Pouriayevali, Y.B. Guo, V.P.W. Shim, A constitutive description of elastomer behaviour at high strain rates - A strain-dependent relaxation time approach, Int J Impact Eng, 47 (2012) 71-78.

[23] R.N. Haward, G. Thackray, The use of a mathematical model to describe isothermal stress-strain curves in glassy thermoplastics, P Roy Soc A-Math Phy, 302 (1968) 453-472. [24] J. Ciambella, A. Paolone, S. Vidoli, A comparison of nonlinear integral-based viscoelastic models through compression tests on filled rubber, Mech Mater, 42 (2010) 932944. 